\title{
Nonlinear Transport Near a Quantum Phase Transition in Two Dimensions
}

\author{
Denis Dalidovich ${ }^{a}$ and Philip Phillips ${ }^{b}$ \\ ${ }^{a}$ National High Field Magnetic Laboratory,Florida State University, Tallahassee, Florida \\ ${ }^{b}$ Loomis Laboratory of Physics, University of Illinois at Urbana-Champaign, 1100 W.Green St., Urbana, IL, 61801-3080
}

\begin{abstract}
The problem of non-linear transport near a quantum phase transition is solved within the Landau theory for the dissipative insulator-superconductor phase transition in two dimensions. Using the nonequilibrium Schwinger round-trip Green function formalism, we obtain the scaling function for the nonlinear conductivity in the quantum disordered regime. We find that the conductivity scales as $E^{2}$ at low field but crosses over at large fields to a universal constant on the order of $e^{2} / h$. The crossover between these two regimes obtains when the length scale for the quantum fluctuations becomes comparable to that of the electric field within logarithmic accuracy.
\end{abstract}

When an electric field is applied to a system near a quantum critical point, non-linear transport obtains. The non-linearity arises because the length scale [1] associated with the electric field scales as $\ell_{E} \propto E^{-1 /(1+z)}$, where $z$ is the dynamical exponent. As it is the ratio of the electric field length scale to the correlation length, $\xi \approx \Delta^{-\nu}$, that enters the resultant DC resistivity,

$$
\rho(T, E)=f\left(\Delta / T^{1 / \nu z}, \Delta / E^{1 / \nu(z+1)}\right),
$$

a non-linear electrical response is inescapable. Here $\Delta$ is the distance from the critical point. Non-linear transport according to Eq. (1) is expected to hold as long as the temperature is low enough so that the length scale associated [7] with temperature, $\ell_{T} \approx 1 / T^{1 / z}$, exceeds that of the electric field, $1 / T^{1 / z} \gg \ell_{E}$. Because of the additional factor of $z$ that enters the electric field scaling contribution to the conductivity, simultaneous scaling of the resistivity with respect to temperature and electric field enables a direct determination of both the correlation and dynamical exponents, $\nu$ and $z$, respectively and hence a complete characterization of the critical properties. Despite its obvious importance, electric field scaling persists as the outstanding problem in quantum criticality because no theoretical account has been put forth to explain how quantum fluctuations conspire to yield non-linear transport. The primary theoretical hurdle is simple: A successful theory of non-linear transport must lie outside the standard Kubo/linear response formalism. Experimentally, the problem is complicated by the fact that in a wide range of systems exhibiting quantum critical points [3-6], the $I-V$ characteristics which are highly non-linear at small fields all become linear at large fields. In fact, the $I-V$ characteristics for varying values of $|\Delta|$ all attain a universal slope as $E \rightarrow \infty$. The vanishing of the non-linearity at high values of $E$ implies that the scaling function for the resistivity is a highly non-trivial and non-monotonic function of the electric field. Hence, a successful theory of non-linear transport must uncloak how such non-monotonicity arises.

Using a non-equilibrium formalism, we calculate ex- plicitly the scaling function for the non-linear conductivity for both the large and small electric field limits. While the formalism can be applied to any critical theory, we focus on the Landau theory for the insulatorsuperconductor transition in which the dynamics are determined by an Ohmic bath; that is, $z=2$. Within the Schwinger [8] round-trip double-time Green function formalism (also known as the Kadanoff-Baym [9], Keldysh formalism [10]), we evaluate the current for arbitrary electric field simply by calculating the appropriate Green function. When temperature is the smallest parameter (quantum disordered regime), a crucial ratio which enters both the conductivity and the inverse correlation length, $m^{2}$, is the ratio

$$
Q=\left(e^{*} E\right)^{2 / 3} / \Delta
$$

$\left(e^{*}=2 e\right.$ - the charge of a Cooper pair $)$. For $Q \ll 1$, corrections due to field are sub-dominant and $\left.m^{2}=\Delta / \ln (1 / \Delta)\right)$ whereas for large field, $m^{2}=$ $\Upsilon\left(e^{*} E\right)^{2 / 3} / \ln \left(1 /\left(e^{*} E\right)^{2 / 3}, \Upsilon\right.$ a constant. In the quantum disordered regime, the static conductivity scales as $Q^{2}$ for $Q \ll 1$, whereas in the large-field limit, $Q \gg$ 1 , linear transport emerges with a universal constant, $\sigma(E)=0.46 e^{2} / h$, that is in qualitative agreement with experiments [3-6].

The starting point for our analysis is the minimal Ginsburg-Landau action (in the imaginary time)

$$
\begin{aligned}
& F[\psi]=\int d^{2} r \int d \tau\left\{\left|\left(\nabla-\frac{i e^{*}}{\hbar} \mathbf{A}(\mathbf{r}, \tau)\right) \psi(\mathbf{r}, \tau)\right|^{2}+\right. \\
& \left.\left|\partial_{\tau} \psi(\mathbf{r}, \tau)\right|^{2}+\delta|\psi(\mathbf{r}, \tau)|^{2}+(U / 2)|\psi(\mathbf{r}, \tau)|^{4}\right\}+L_{\text {dis }}
\end{aligned}
$$

required to model quantum fluctuations and dissipation near the zero-resistance quantum critical point. In Eq. (3), $\mathbf{A}(\mathbf{r}, \tau)$ is the vector potential, $e^{*}=2 e, \delta$ is the bare distance to the quantum critical point. In terms of the Matsubara frequencies, the dissipation term, $L_{\mathrm{dis}}=\eta \sum_{\mathbf{k}, \omega_{n}}\left|\omega_{n} \| \psi\left(\mathbf{k}, \omega_{n}\right)\right|^{2}$, corresponds to the phenomenological Ohmic model introduced by Caldeira and Leggett [11], in which $\eta$ measures the strength of the dissipation. The order parameter $\psi(\mathbf{r}, \tau)$ is the standard 
two-component complex field whose expectation value is non-zero in the superconducting phase. Our motivation for introducing dissipation, that is expected to be strong $(\eta \sim 1)$, is two-fold. First, the second-derivative term now becomes irrelevant. Consequently, the zero temperature transition falls into the $z=2$ universality class. Such a universality class describes a 2D insulator to high$T_{c}$ superconductor transition in which superconductivity is destroyed by impurities [12]. Second, as we will see, $z=2$ dynamics are inherently easier to solve using the Schwinger $[10,13]$ technique than is the corresponding $z=1$ problem. Nonetheless, the generality of our conclusions leads us to believe that similar results must hold for the $z=1$ case as well.

As the problem we wish to treat is inherently out of equilibrium, we resort to the real-time 'round-trip' Schwinger formalism $[8-10,13]$ which is ideally suited for solving problems in which an asymmetry exists between forward and backward evolution in time. We will be brief in our presentation of this technique as it is welldocumented in the literature $[10,13,14]$ and our notation will follow that of Lifshitz and Pitaevskii [13]. To use the real-time formalism, one needs to rewrite the action, Eq. (3, as an integral over the Keldysh contour. A constant in space and time electric field is assumed to enter the action via the vector potential, $\mathbf{A}(t)=-\mathbf{E} t$. This gauge choice allows for all functions to be Fourier expandable in space, since full translational invariance is retained. We define the time ordered and anti-time ordered Green functions

$$
\begin{aligned}
& i G_{\mathbf{p}}^{--}\left(t_{1}, t_{2}\right)=F T\left\langle T\left[\psi\left(\mathbf{r}, t_{1}\right), \psi^{*}\left(\mathbf{r}^{\prime}, t_{2}\right)\right]\right\rangle \\
& i G_{\mathbf{p}}^{++}\left(t_{1}, t_{2}\right)=F T\left\langle\left[\tilde{T} \psi\left(\mathbf{r}, t_{1}\right), \psi^{*}\left(\mathbf{r}^{\prime}, t_{2}\right)\right]\right\rangle,
\end{aligned}
$$

where $T$ and $\tilde{T}$ are the time ordering and inverse time ordering operators. Here FT represents the Fourier transform with respect to space. We will need also the nontime ordered functions

$$
\begin{aligned}
& i G_{\mathbf{p}}^{-+}\left(t_{1}, t_{2}\right)=F T\left\langle\psi^{*}\left(\mathbf{r}^{\prime}, t_{2}\right) \psi\left(\mathbf{r}, t_{1}\right)\right\rangle \\
& i G_{\mathbf{p}}^{+-}\left(t_{1}, t_{2}\right)=F T\left\langle\psi\left(\mathbf{r}, t_{1}\right) \psi^{*}\left(\mathbf{r}^{\prime}, t_{2}\right)\right\rangle
\end{aligned}
$$

the last of which is directly related to the expectation value of the current operator through

$$
\mathbf{J}(t)=\frac{-2 i e^{*}}{\hbar} \int \mathbf{p} \frac{d^{d} p}{(2 \pi)^{d}} G_{\mathbf{p}}^{-+}(t, t)
$$

The average values in these expressions are over all states of the system, not simply equilibrium ones. Since the four functions in Eqs. (4) and (5) forming a 2 by 2 matrix $\hat{G}$, are not independent, one employs the so-called Keldysh rotation to work with the three independent functions $G^{R}\left(G^{A}\right)=G^{--}-G^{-+}\left(G^{+-}\right), G^{K}=G^{-+}+G^{+-}$. The matrix of the exact Green functions $\hat{G}$ is connected to that of the Green functions for free quasi-particles $\hat{G}^{(0)}$ via the Dyson equation [13], $\hat{G}=\hat{G}^{(0)}+\iint \hat{G}^{(0)} \hat{\Sigma} \hat{G}$, in which the integration over internal time and space arguments is assumed and the self-energy matrix $\hat{\Sigma}$ is itself in general a complicated functional of $\hat{G}$. For the static field, the resulting Green functions $G^{+-}, G^{-+}, G^{K}$, taken at $t=t_{1}=t_{2}$ do not depend on $t$, reflecting the fact that the system is time translationally invariant as well.

The non-linear conductivity is defined as the constant of proportionality between the current and the electric field. We will orient the field along $\mathrm{x}$-axis and hence $J_{x x}(E)=\sigma_{x x}(E) E$. In the Schwinger formalism, the direct relationship [14] between $G^{-+}$and the retarded and advanced Green functions

$G_{\mathbf{p}}^{-+}\left(t_{1}, t_{2}\right)=-\int d t_{3} d t_{4} G_{\mathbf{p}}^{R}\left(t_{1}, t_{3}\right) \Sigma^{-+}\left(t_{3}, t_{4}\right) G_{\mathbf{p}}^{A}\left(t_{4}, t_{2}\right)$

is obtained by formally solving the Dyson equation. In Eq. (7), note the minus sign that is absent in the fermion problem [14]. Analogous expressions hold for $G^{+-}\left(G^{K}\right)$ with $\Sigma^{-+}$replaced with $\Sigma^{+-}\left(\Sigma^{K}\right)$. In general, the selfenergy $\hat{\Sigma}$ arises from the interaction $U, \hat{\Sigma}_{U}$, and the coupling to the dissipative bath $\hat{\Sigma}_{d}$. However, treating the interactions in the large- $\mathrm{N}$ (mean-field) limit, we obtain that this approximation gives rise only to the renormalization of the bare distance to the critical point in the action [15],

$$
m^{2}=\delta-\frac{U}{2} \int \frac{d^{2} p}{(2 \pi)^{2}} i G^{K}(p, t, t),
$$

so that a new Gaussian action with the excitation spectrum $\epsilon(\mathbf{p})=\mathbf{p}^{2}+m^{2}$ obtains. There is no contribution from interactions to $\Sigma^{-+}$(and $\Sigma^{K}$ ) at this level, and the corresponding Green functions are assessed by substituting $\Sigma_{d}\left(t_{3}-t_{4}\right)$ into Eq. (7). To this end, we simplify the notation by setting $\eta$ equal to unity, so that in the frequency space $[15] \Sigma_{d}^{-+}(\omega)=$ $-2 i \omega e^{-|\omega| / \Lambda} n_{B}(\omega), \quad \Sigma_{d}^{R}(\omega)=\Sigma^{A}(\omega)^{*}=i \omega e^{-|\omega| / \Lambda}$, $\Sigma_{d}^{K}(\omega)=2 i \omega e^{-|\omega| / \Lambda}\left(2 n_{B}(\omega)+1\right)$, with $n_{B}(\omega)$ the distribution function for bosons. The upper frequency cutoff $\Lambda$ is necessary only to ensure the convergence of the zero temperature parts of the Fourier integrals $\Sigma_{d}(t)=$ $\int \Sigma_{d}(\omega)(d \omega / 2 \pi) e^{-i \omega t}$.

The equation of motion for the corresponding retarded Green function

$$
\frac{\partial}{\partial t_{1}} G_{\mathbf{p}}^{R}\left(t_{1}, t_{2}\right)+\epsilon\left(\mathbf{p}-e^{*} \mathbf{E} t_{1}\right) G_{\mathbf{p}}^{R}\left(t_{1}, t_{2}\right)=\delta\left(t_{1}-t_{2}\right)
$$

has a simple solution

$$
G_{\mathbf{p}}^{R}\left(t_{1}, t_{2}\right)=\theta\left(t_{1}-t_{2}\right) \exp \left\{-\int_{t_{1}}^{t_{2}} \epsilon\left(\mathbf{p}-e^{*} \mathbf{E} \tau\right) d \tau\right\} .
$$

In $G^{A}$, the order of the time arguments is reversed in the step function $\theta(x)$. Consequently, after Eqs. (7), and (10) are combined, our problem is, in principle, solved. 
To make contact with the case $E=0$, we note that in the absence of a field, the frequency Fourier transform of Eq. (10) reduces to the expected result $(\epsilon(\mathbf{p})-i \eta \omega)^{-1}$.

When the field is non-zero, two distinct regimes emerge. To obtain an estimate of the crossover field, we determine at which value of the field can the exponential in $G^{R}$ be expanded. We rewrite $\epsilon(\mathbf{p})$ in the exponential of Eq. (10) as $p^{2}+2 p_{x} e^{*} E \tau+m^{2}$. Upon integrating the resultant exponential in Eq. (10), we find that the electric-field dependence can be removed from the exponential provided that $p_{x} e^{*} E t^{2} \ll 1$. Since both momenta and time are integrated over, this criterion is never satisfied. Hence, strictly speaking, the electric field effects are always non-perturbative. Nonetheless, if we focus on the characteristic timescale, $t \propto 1 / m^{2}$ and momentum scale, $p_{x} \propto m$, we find that $e^{*} E / m^{3}<<1$ or equivalently, $\left(e^{*} E\right)^{2 / 3} / m^{2}=Q \ll 1$ determines the small and large field regimes. It is this criterion, rather than $1 / T^{1 / z} \gg \ell_{E}$, which will serve to determine when nonlinear transport according to Eq.(1) holds.

To obtain the conductivity, we need first calculate $G^{-+}$. We perform the integration in the exponent of Eq. (10) and switch to the Wigner coordinates [14,13]: $t=t_{1}-t_{2}$ and $u=\left(t_{1}+t_{2}\right) / 2$. The Green function from which the current is obtained reduces to

$$
-i G_{\mathbf{p}}^{-+}(t, t)=\int_{0}^{\infty} d u \int_{-2 u}^{2 u} d t M\left(\mathbf{p}, e^{*} E ;, u, t\right) K(t)
$$

where $K(t)=-i \Sigma^{-+}(-t)$ and

$$
\begin{aligned}
M\left(\mathbf{p}, e^{*} E ; u, t\right)= & \exp \left\{-\left(2 \epsilon(\mathbf{p}) u-2 p_{x} e^{*} E\left(2 u^{2}+t^{2} / 2\right)\right.\right. \\
& \left.\left.+\frac{\left(e^{*} E\right)^{2}}{3}\left(u^{3}+3 u t^{2} / 2\right)\right)\right\}
\end{aligned}
$$

and the kernel $K(t)$ is obtained as a result of the appropriate integration over $\omega$ with the cutoff $\Lambda$ necessary to regularize the $T=0$ part. The result is

$$
\begin{aligned}
K(t) & =\frac{1}{\pi} \frac{\Lambda^{-2}-t^{2}}{\left(\Lambda^{-2}+t^{2}\right)^{2}}+\frac{1}{\pi}\left[\frac{1}{t^{2}}-\frac{\pi^{2} T^{2}}{\sinh ^{2}(\pi T t)}\right] \\
& =K_{0}(t)+K_{T}(t)
\end{aligned}
$$

in which the second temperature dependent part $K_{T}(t) \approx$ $\pi T^{2} / 3$ as $T t \ll 1$. Similarly, because $G^{K}=G^{-+}+G^{+-}$, we can also express the inverse correlation length

$$
m^{2}=\delta+U \int_{\mathbf{p}} \int_{0}^{\infty} d u \int_{-2 u}^{2 u} d t M\left(\mathbf{p}, e^{*} E ; u, t\right) K(t)
$$

in terms of the same functions appearing in $G^{-+}$.

Our problem has been reduced then to the computation of two types of coupled integrals in Eqs. (14) and (11). In the $T \rightarrow 0$ limit, the main difficulty is to obtain the cutoff-free expressions for $m$ and $J$, since the physical results should obey the universal scaling and correspond to $\Lambda^{-1}=0$. In this regard, we have found the following procedure most helpful: 1) Regularize the zero-temperature part (that is, the integrals over the product $\left.M\left(\mathbf{p}, e^{*} E ; u, t\right) K_{0}(t)\right)$ by adding and subtracting $M\left(\mathbf{p}, e^{*} E ; u, 0\right)$ and $M(\mathbf{p}, 0 ; u, 0)$ to $M\left(\mathbf{p}, e^{*} E ; u, t\right) K_{0}(t)$. In the current, the integral over $\mathbf{p}$ in the part involving $M(\mathbf{p}, 0 ; u, 0)$ vanishes identically. In Eq. (11) similar contribution gives rise to the frequency and momentum dependent renormalizations of $\delta$, as well as the term $m^{2} \ln \left(1 / m^{2}\right)$, ensuring the validity of the subsequently used logarithmic accuracy. 2) In the part containing $M\left(\mathbf{p}, e^{*} E ; u, 0\right)-M(\mathbf{p}, 0 ; u, 0)$, perform straightforwardly the integration over $t$ in $K_{0}(t)$ to yield $2 /(u \pi) .3)$ In the part containing $M\left(\mathbf{p}, e^{*} E ; u, t\right)-$ $M\left(\mathbf{p}, e^{*} E ; u, 0\right)$, set $\Lambda^{-1}=0$ as this term is completely convergent. 4) In the terms containing the aforementioned differences, we introduce the change of variables $p_{x} \rightarrow p_{x}-\left(e^{*} E\right) u / 2\left(1+t^{2} / 4 u^{2}\right)$, and perform the Gaussian integration over momenta $p_{x}$ and $p_{y}$. Similar integration is performed in the part containing $K_{T}$. 5) Finally, change variables to $y=t / 2 u$ and $z=u\left(e^{*} E\right)^{2 / 3}$. These transformations lead to the appearance of the auxilliary functions

$$
\begin{aligned}
& M_{2}(z)=\exp \left\{-\frac{2 m^{2} z}{\left(e^{*} E\right)^{2 / 3}}\right\} \\
& M_{1}(z, y)=M_{2}(z) \exp \left\{-z^{3}\left(1 / 6+y^{2}-y^{4} / 2\right)\right\}
\end{aligned}
$$

and $\tilde{K}_{T}(z, y)=K_{T}\left(t=2 z\left(e^{*} E\right)^{4 / 3} y\right)$. The expressions determining the current reduces now to quadrature:

$$
\begin{aligned}
J= & \frac{2 e^{*}\left(e^{*} E\right)}{16 \pi^{2} \hbar}\left\{\int_{0}^{\infty} \frac{d z}{z} \int_{0}^{1} \frac{d y}{y^{2}} f(y)\left[M_{1}(z, 0)-M_{1}(z, y)\right]\right. \\
& \left.+\frac{4 \pi}{\left(e^{*} E\right)^{4 / 3}} \int_{0}^{\infty} z d z \int_{0}^{1} d y \tilde{K}_{T}(z, y) f(y) M_{1}(z, y)\right\}
\end{aligned}
$$

with $f(y)=\left(1+y^{2}\right)$. Similarly, within logarithmic accuracy, we have

$$
\begin{aligned}
& m^{2} \ln \left(1 / m^{2}\right)=\Delta+\left(e^{*} E\right)^{2 / 3}\left\{\int _ { 0 } ^ { \infty } \frac { d z } { 2 z ^ { 2 } } \int _ { 0 } ^ { 1 } \frac { d y } { y ^ { 2 } } \left[M_{1}(z, 0)\right.\right. \\
& \left.-M_{1}(z, y)\right]-\int_{0}^{\infty} \frac{d z}{2 z^{2}}\left[M_{2}(z)-M_{1}(z, 0)\right] \\
& \left.+\frac{2 \pi}{\left(e^{*} E\right)^{4 / 3}} \int_{0}^{\infty} d z \int_{0}^{1} d y \tilde{K}_{T}(z, y) M_{1}(z, y)\right\}
\end{aligned}
$$

where $\Delta$ is the renormalized distance to the quantum critical point.

Consider now the quantum-disordered regime, in which $T$ is the smallest parameter. At low electric fields, $Q=\left(e^{*} E\right)^{2 / 3} / \Delta \ll 1$, we expand Eqs. (17) and (16) in powers of $Q$ to obtain

$$
m^{2}=\frac{\Delta}{\ln (1 / \Delta)}+\frac{\left(e^{*} E\right)^{2}}{12\left(\Delta^{2} / \ln (1 / \Delta)\right)}+\frac{\pi^{2}}{3} \frac{T^{2}}{\Delta / \ln (1 / \Delta)}
$$

and 


$$
J=\frac{4 e^{2}}{h}\left[\frac{\pi T^{2}}{9 m^{4}}+\frac{\left.\left(e^{*} E\right)^{2}\right)}{15 \pi m^{6}}\right] E=\sigma(E) E, \quad Q \ll 1 .
$$

for the inverse correlation length and the non-linear current-voltage response. The first term matches identically with the value of the linear current response calculated earlier [16] for the quantum disordered regime. Most importantly, the current scales as the third power of the voltage in the quantum disordered regime. This result clearly could not have been obtained within linear response theory. This non-linear response gives rise to a non-zero conductivity (albeit non-linear) even at $T=0$ on the putative insulating side of the transition. Such a term arises entirely from the zero-temperature part of the integrals in Eq. (16). As such a term will always be present regardless of which universality class describes the quantum phase transition, we predict that the $T=0$ non-linear conductivity will always remain non-zero on the disordered side of the transition. This conclusion is borne out experimentally by the series of measurements of $d I / d V$ in insulator-superconductor [3,4], quantum Hall to insulator [5], and insulator to metal transitions [6]. Further, the form of the non-linearity, $E^{3}$, is non-trivial and is also in agreement with the pronounced curvature in the $I-V$ curves on the insulating side of the dissipative insulator-superconductor transition [4]. To explore the large field regime from Eq. (16), we note that when $Q \gg 1, e^{*} E$ is the only parameter that determines $m$. Anticipating the logarithmic smallness, we set $M_{2}(z) \approx 1$, and after performing first the integral over $z$, we obtain

$$
m^{2}=\frac{\Upsilon\left(e^{*} E\right)^{2 / 3}}{\ln \left(1 /\left(e^{*} E\right)^{2 / 3}\right)},
$$

where

$$
\begin{aligned}
\Upsilon= & \frac{6^{2 / 3}}{12} \Gamma\left(\frac{2}{3}\right) \int_{0}^{1}\left[\frac{\left(1+6 y^{2}-3 y^{4}\right)^{1 / 3}-f(y)}{y^{2}}\right] d y \\
= & 0.116525 .
\end{aligned}
$$

Here, as before, $f(y)=1+y^{2}$. In the same limit, the current reduces to a single integral of the form,

$$
\begin{aligned}
J= & \frac{2 e^{*}}{4 \pi^{2} \hbar} \frac{e^{*} E}{12} \int_{0}^{1} \frac{d y}{y^{2}} f(y) \ln \left(1+6 y^{2}-3 y^{4}\right) \\
& =0.46 \frac{e^{2}}{h} E \quad Q \gg 1
\end{aligned}
$$

which proves that $d I / d V$ approaches a universal constant, which of course depends on the universality class, as $E \rightarrow \infty$. At present, the most we can conclude is that the constant is of order $e^{2} / h$. This is expected to be of order $e^{2} / h$ as it should be on the order of the normal sheet resistance. Hence, we have developed a formalism which is capable of describing the experimental crossover from the non-linear to the linear regime at high field.
The crossover to the linear regime has a fundamental origin. Because at mean-field $\nu=1 / 2$ and $z=2$, we can rewrite Eq. (20) as $m^{2} \propto 1 /\left(\ell_{E}^{1 / \nu} \ln \left(\ell_{E}^{1 / \nu}\right)\right)$. Hence, at large fields, the correlation length is cutoff by the electric field length scale, to logarithmic accuracy. That is, the only length scale in the large field limit is $\ell_{E}$. As it is the product $\left(m \ell_{E}\right)^{\nu}$ that enters the non-linear conductivity, the electric field dependence naturally drops out and the conductivity approaches a universal constant. In the opposite regime, the correlation length and the electric field length scale are distinct as Eq. (18) indicates. The non-linearity arises entirely from the quantum fluctuations on the length scale $\xi$. In the non-linear regime, it is tempting to rewrite the current, Eq. (19), in terms of an effective temperature, $T_{\text {eff }}^{2}=T^{2}+0.06\left(e^{*} E / m\right)^{2}$. The current then simplifies to

$$
J=\frac{4 \pi e^{*}}{9 h} T_{\text {eff }}^{2} E .
$$

For electrons localized in the band tails of semiconductors, the concept of an electric field-dependent effective temperature has been used extensively $[18,19]$. Electrons moving against the electric field are accelerated on a length scale set by the localization length. In the quantum disordered regime, it is the correlation length that plays the role of the localization length. Consequently, it is not surprising that the bosonic excitations can be described by an effective E-field dependent effective temperature.

This work was partially funded by donors of the ACS petroleum research fund and the NSF DMR-0305864 .

[1] M. P. A. Fisher, Phys. Rev. Lett. 65, 923 (1990).

[2] S. Sondhi et. al., Rev. Mod. Phys. 69, 315 (1997).

[3] A. Yazdani and A. Kapitulnik, Phys. Rev. Lett. 74, 30373040 (1995).

[4] A. J. Rimberg, et. al., Phys. Rev. Lett. 78, 2632 (1997).

[5] D. Shahar, et. al., Phys. Rev. Lett. 74, 4511 (1995).

[6] S. V. Kravchenko, et. al., Phys. Rev. Lett. 77, 4938 (1996).

[7] S. Sondhi et. al., Rev. Mod. Phys. 69, 315 (1997).

[8] J. Schwinger, J. Math. Phys. 2, 407 (1961).

[9] L. P. Kadanoff and G. Baym, Quantum Statistical Mechanics, (W. A. Benjamin, Inc. 1962).

[10] L. V. Keldysh, Sov. Phys. JETP 20, 1018 (1965).

[11] A. O. Caldeira and A. J. Leggett, Phys. Rev. Lett. 46, 211 (1981).

[12] I. F. Herbut, Phys. Rev. Lett. 85, 1532 (2000).

[13] E. M. Lifshitz and L. P. Pitaevskii, Physical Kinetics, (Pergamon Press, Oxford, 1981) Chap 10.

[14] F. S. Khan, J. H. Davies, and J. W. Wilkins, Phys. Rev. B 36, 2578 (1987). 
[15] M. P. Kennett, C. Chamon and J. Ye, Phys. Rev. B 64, 224408 (2001).

[16] D. Dalidovich and P. Phillips, Phys. Rev. Lett. 84, 737 (2000).

[17] S. Sachdev, A. Chubukov, and S. Sokol, Phys. Rev. B 51, 14874 (1995).

[18] S. Marianer and B. I. Shklovskii, Phys. Rev. B 46, 13100 (1992).

[19] P. Phillips, Phys. Rev. B 49, 4303 (1994). 